\title{
Clinical and translational imaging: on a wave of progressive development
}

\author{
Giovanni Lucignani ${ }^{1,2}$
}

Published online: 16 December 2016

(C) Italian Association of Nuclear Medicine and Molecular Imaging 2016

Clinical and Translational Imaging (CATI) is embarking upon its 5th year of life on a wave of progressive development. Thanks to our authors, to the outstanding work of our colleagues acting as editors and referees, to the support of the Italian Association of Nuclear Medicine and to the skillful assistance of the Springer team, during the past year we have achieved new growth records.

Thanks to coordinated teamwork during the past 12 months we have published series of papers dealing with dose optimization [1], pediatric imaging [2], in vivo imaging of microorganisms [3], radioembolization [4], radioguided intervention [5], and prostate cancer [6]. Many other papers on other topics were also accepted.

The goals set in 2015 and 2016 were achieved, and the number of high-quality papers being submitted clearly shows the regard in which our journal is held by clinicians and scientists. Moreover, the numbers of downloads and citations are also increasing steeply, faster than the number of published papers.

Analysis of the main bibliometric indices at the end of 2016 indicates an h-index of 11 for CATI (compared with 4 in 2013, 7 in 2014 and 9 in 2016) while the ratio of published papers to citing articles, a surrogate indicator of the impact factor, is also increasing steadily.

Giovanni Lucignani

giovanni.lucignani@unimi.it

1 Department of Health Sciences (DISS) and Center of Molecular and Cellular Imaging (IMAGO), University of Milan, Milan, Italy

2 Unit of Nuclear Medicine, Department of Diagnostic Services, ASST Santi Paolo e Carlo, Via Antonio di Rudinì 8, 20142 Milan, Italy
In relation to this growth, I am also happy to report that after a first selective step in 2015 leading to inclusion in Scopus, CATI is now included also in the Emerging Sources Citation Index (ESCI) database, a new Thomson Reuters product: "ESCI extends the universe of publications in Web of Science to include high-quality, peer-reviewed publications of regional importance and in emerging scientific fields. ESCI will also make content important to funders, key opinion leaders, and evaluators visible in Web of Science Core Collection even if it has not yet demonstrated citation impact on an international audience."

In other words, in only its 4th year of existence our journal has passed a further selective assessment, this time preliminary selection for inclusion in Journal Citation Reports, the exclusive club of publications with an official impact factor. It is worth recalling, however, for those interested in bibliometric indexing with respect to the assessment of their academic achievements and grant applications, that papers and citations in journals indexed in Scopus are valued in many countries worldwide.

Since CATI is now also accepting papers that are not solicited within the series promoted by guest editors, a number of papers on diverse and heterogeneous topics have been published during the last year, covering aspects of radiochemistry, radiation dosimetry, and clinical themes. More such papers are expected, and our entire readership is welcome to contribute.

During this year we have also enhanced our visibility by opening Facebook (https://www.facebook.com/Clinicaland TranslationalImaging/) and Twitter (https://twitter.com/ CAT_Imaging) accounts and by posting news about journal activity in various Facebook groups aimed at scientists and clinicians involved in nuclear medicine and molecular imaging, thus reaching a broad yet focused international 
readership via this new route. The number of article downloads has increased fourfold and the number of contacts tenfold. In this way we aim to create a nuclear medicine scientific community around the journal and promote discussions on new, original or controversial contents to further increase clinical research in the field.

I would like to remind our readers that, upon payment of a fee, CATI offers the option of Open Access publication, resulting in immediate inclusion in PubMed, the search engine that ensures worldwide visibility. Furthermore a new Springer policy allows authors to share their published article by means of the Springer Nature SharedIt link. To assist authors in disseminating their research to the broader community, Springer provides a free read-only version of the full published article via a unique SharedIt link that can be generated by entering the DOI of the article.

During the past year we have also slightly reorganized the classification of accepted papers. Reviews are now divided into four categories-Systematic Reviews, MetaAnalyses, Expert Reviews, and Pictorial Reviews-as described in the Instructions for Authors. Finally, in order to be more informative for its readers the journal now requires structured abstracts describing the purpose, methods, results and conclusions of each paper.
Our priorities include progressively higher visibility for our content and continuing to be informative for our community, with the contribution of all those interested in the progress of our discipline.

\section{References}

1. Lassmann M, Pedroli G (2016) Dose optimization in nuclear medicine. Clin Transl Imaging 4:3. doi:10.1007/s40336-015-0154-7

2. De Palma D, Mansi L, Treves ST (2016) Pediatric molecular imaging today: new technologies, new challenges, new answers. Clin Transl Imaging 4:75. doi:10.1007/s40336-015-0157-4

3. Signore A, Santino I, Glaudemans AWJM (2016) In vivo imaging of microorganisms. Clin Transl Imaging 4:161. doi:10.1007/ s40336-016-0176-9

4. Maccauro M, Salem R (2016) The impact of clinical factor on dosimetry in radioembolisation. Clin Transl Imaging 4:225. doi:10.1007/s40336-016-0188-5

5. Valdés Olmos RA, Vidal-Sicart S (2016) Introducing new perspectives in radioguided intervention. Clin Transl Imaging 4:307. doi:10.1007/s40336-016-0177-8

6. Fanti S, Giammarile F (2016) Molecular imaging and prostate cancer: unmet clinical needs and future perspectives. Clin Transl Imaging 4:421. doi:10.1007/s40336-016-0210-y 